\title{
Observation of a Resonant Structure in $e^{+} e^{-} \rightarrow K^{+} K^{-} \pi^{0} \pi^{0}$
}

M. Ablikim, ${ }^{1}$ M. N. Achasov, ${ }^{10, e}$ P. Adlarson, ${ }^{63}$ S. Ahmed, ${ }^{15}$ M. Albrecht, ${ }^{4}$ A. Amoroso, ${ }^{62 a, 62 c}$ Q. An,${ }^{59,47}$ Anita, ${ }^{21}$ Y. Bai,${ }^{46}$ O. Bakina, ${ }^{28}$ R. Baldini Ferroli, ${ }^{23 a}$ I. Balossino, ${ }^{24 a}$ Y. Ban, ${ }^{37, m}$ K. Begzsuren, ${ }^{26}$ J. V. Bennett, ${ }^{5}$ N. Berger, ${ }^{27}$ M. Bertani, ${ }^{23 a}$ D. Bettoni, ${ }^{24 a}$ F. Bianchi, ${ }^{62,62 \mathrm{c}}$ J. Biernat, ${ }^{63} \mathrm{~J}$. Bloms, ${ }^{56}$ I. Boyko, ${ }^{28}$ R. A. Briere, ${ }^{5} \mathrm{H} . \mathrm{Cai},{ }^{64} \mathrm{X}$. Cai, ${ }^{1,47}$ A. Calcaterra, ${ }^{23 \mathrm{a}}$ G. F. Cao, ${ }^{1,51}$ N. Cao, ${ }^{1,51}$ S. A. Cetin,${ }^{50 b}$ J. F. Chang, ${ }^{1,47}$ W. L. Chang,,${ }^{1,51}$ G. Chelkov, ${ }^{28, c, d}$ D. Y. Chen, ${ }^{6}$ G. Chen, ${ }^{1}$ H. S. Chen, ${ }^{1,51}$ M. L. Chen, ${ }^{1,47}$ S. J. Chen, ${ }^{35}$ X. R. Chen, ${ }^{25}$ Y. B. Chen, ${ }^{1,47}$ W. Cheng, ${ }^{62 \mathrm{c}}$ G. Cibinetto, ${ }^{24 a}$ F. Cossio, ${ }^{62 \mathrm{c}}$ X. F. Cui, ${ }^{36}$ H. L. Dai,${ }^{1,47}$ J. P. Dai, ${ }^{41, i}$ X. C. Dai, ${ }^{1,51}$ A. Dbeyssi, ${ }^{15}$ D. Dedovich,${ }^{28}$ Z. Y. Deng, ${ }^{1}$ A. Denig, ${ }^{27}$ I. Denysenko, ${ }^{28}$ M. Destefanis, ${ }^{62 a, 62 c}$ F. De Mori, ${ }^{62 a, 62 c}$ Y. Ding, ${ }^{33}$ C. Dong, ${ }^{36}$ J. Dong, ${ }^{1,47}$ L. Y. Dong, ${ }^{1,51}$ M. Y. Dong, ${ }^{1,47,51}$ S. X. Du, ${ }^{67}$ J. Fang, ${ }^{1,47}$ S. S. Fang, ${ }^{1,51}$ Y. Fang, ${ }^{1}$ R. Farinelli, ${ }^{24 a, 24 b}$ L. Fava, ${ }^{62 b, 62 c}$ F. Feldbauer, ${ }^{4}$ G. Felici, ${ }^{23 a}$ C. Q. Feng, ${ }^{59,47}$ M. Fritsch, ${ }^{4}$ C. D. Fu, ${ }^{1}$ Y. Fu, ${ }^{1}$ X. L. Gao,${ }^{59,47}$ Y. Gao, ${ }^{37, \mathrm{~m}}$ Y. Gao, ${ }^{60}$ Y. G. Gao, ${ }^{6}$ I. Garzia, ${ }^{24 a, 24 b}$ E. M. Gersabeck, ${ }^{54}$ A. Gilman, ${ }^{55}$ K. Goetzen, ${ }^{11}$ L. Gong, ${ }^{36}$ W. X. Gong, ${ }^{1,47}$ W. Gradl, ${ }^{27}$ M. Greco, ${ }^{62 a, 62 c}$ L. M. Gu, ${ }^{35}$ M. H. Gu, ${ }^{1,47}$ S. Gu, ${ }^{2}$ Y. T. Gu, ${ }^{13}$ C. Y. Guan, ${ }^{1,51}$ A. Q. Guo, ${ }^{22}$ L. B. Guo ${ }^{34}$ R. P. Guo, ${ }^{39}$ Y. P. Guo,${ }^{27}$ Y. P. Guo, ${ }^{9, j}$ A. Guskov, ${ }^{28}$ S. Han,${ }^{64}$ T. T. Han, ${ }^{40}$ T. Z. Han, ${ }^{9, j}$ X. Q. Hao, ${ }^{16}$ F. A. Harris, ${ }^{52}$ K. L. He ${ }^{1,51}$ F. H. Heinsius, ${ }^{4}$ T. Held, ${ }^{4}$ Y. K. Heng, ${ }^{1,47,51}$ M. Himmelreich, ${ }^{11, h}$ T. Holtmann, ${ }^{4}$ Y. R. Hou,${ }^{51}$ Z. L. Hou, ${ }^{1}$ H. M. Hu, ${ }^{1,51}$ J. F. Hu,${ }^{41, i}$ T. Hu, ${ }^{1,47,51}$ Y. Hu, ${ }^{1}$ G. S. Huang, ${ }^{59,47}$ L. Q. Huang, ${ }^{60}$ X. T. Huang, ${ }^{40}$ N. Huesken, ${ }^{56}$ T. Hussain,${ }^{61}$ W. Ikegami Andersson, ${ }^{63}$ W. Imoehl,${ }^{22}$ M. Irshad,${ }^{59,47}$ S. Jaeger, ${ }^{4}$ S. Janchiv, $, 6,1$ Q. Ji, ${ }^{1}$ Q. P. Ji, ${ }^{16}$ X. B. Ji, ${ }^{1,51}$ X. L. Ji, ${ }^{1,47}$ H. B. Jiang, ${ }^{40}$ X. S. Jiang, ${ }^{1,47,51}$ X. Y. Jiang, ${ }^{36}$ J. B. Jiao,${ }^{40}$ Z. Jiao, ${ }^{18}$ D. P. Jin, ${ }^{1,47,51}$ S. Jin, ${ }^{35}$ Y. Jin, ${ }^{53}$ T. Johansson, ${ }^{63}$ N. Kalantar-Nayestanaki, ${ }^{30}$ X. S. Kang, ${ }^{33}$ R. Kappert, ${ }^{30}$ M. Kavatsyuk, ${ }^{30}$ B. C. Ke, ${ }^{42,1}$ I. K. Keshk, ${ }^{4}$ A. Khoukaz, ${ }^{56}$ P. Kiese, ${ }^{27}$ R. Kiuchi, ${ }^{1}$ R. Kliemt, ${ }^{11}$ L. Koch, ${ }^{29}$ O. B. Kolcu, ${ }^{50 b, g}$ B. Kopf, ${ }^{4}$ M. Kuemmel, ${ }^{4}$ M. Kuessner, ${ }^{4}$ A. Kupsc,${ }^{63}$ M. G. Kurth, ${ }^{1,51}$ W. Kühn, ${ }^{29}$ J. J. Lane, ${ }^{54}$ J. S. Lange, ${ }^{29}$ P. Larin, ${ }^{15}$ L. Lavezzi, ${ }^{62 c}$ H. Leithoff, ${ }^{27}$ M. Lellmann, ${ }^{27}$ T. Lenz, ${ }^{27}$ C. Li ${ }^{38}$ C. H. Li,${ }^{32}$ Cheng Li,${ }^{59,47}$ D. M. Li,${ }^{67}$ F. Li,${ }^{1,47}$ G. Li, ${ }^{1}$ H. B. Li, ${ }^{1,51}$ H. J. Li, ${ }^{9, j}$ J. C. Li, ${ }^{1}$ J. L. Li ${ }^{40}{ }^{K e ~ L i},{ }^{1}$ L. K. Li, ${ }^{1}$ Lei Li, ${ }^{3}$ P. L. Li,${ }^{59,47}$ P. R. Li, ${ }^{31}$ S. Y. Li, ${ }^{49}$ W. D. Li, ${ }^{1,51}$ W. G. Li, ${ }^{1}$ X. H. Li, ${ }^{59,47}$ X. L. Li, ${ }^{40}$ X. N. Li ${ }^{1,47}$ Z. B. Li ${ }^{48}$ Z. Y. Li, ${ }^{48}$ H. Liang, ${ }^{1,51}$ H. Liang, ${ }^{59,47}$ Y. F. Liang, ${ }^{44}$ Y. T. Liang, ${ }^{25}$ L. Z. Liao, ${ }^{1,51}$ J. Libby, ${ }^{21}$ C. X. Lin ${ }^{48}$ D. X. Lin ${ }^{15}$ B. Liu ${ }^{41, i}$ B. J. Liu, ${ }^{1}$ C. X. Liu, ${ }^{1}$ D. Liu,${ }^{59,47}$ D. Y. Liu,${ }^{41, i}$ F. H. Liu,${ }^{43}$ Fang Liu, ${ }^{1}$ Feng Liu, ${ }^{6}$ H. B. Liu, ${ }^{13}$ H. M. Liu, ${ }^{1,51}$ Huanhuan Liu, ${ }^{1}$ Huihui Liu, ${ }^{17}$ J. B. Liu, ${ }^{59,47}$ J. Y. Liu, ${ }^{1,51}$ K. Liu, ${ }^{1}$ K. Y. Liu, ${ }^{33}$ Ke Liu, ${ }^{6}$ L. Liu,,${ }^{59,47}$ L. Y. Liu, ${ }^{13}$ Q. Liu, ${ }^{51}$ S. B. Liu, ${ }^{59,47}$ Shuai Liu, ${ }^{45}$ T. Liu, ${ }^{1,51}$ X. Liu, ${ }^{31}$ X. Y. Liu, ${ }^{1,51}$ Y. B. Liu, ${ }^{36}$ Z. A. Liu, ${ }^{1,47,51}$ Z. Q. Liu, ${ }^{40}$ Y. F. Long, ${ }^{37, m}$ X. C. Lou, ${ }^{1,47,51}$ H. J. Lu, ${ }^{18}$ J. D. Lu, ${ }^{1,51}$ J. G. Lu ${ }^{1,47}$ X. L. Lu, ${ }^{1}$ Y. Lu, ${ }^{1}$ Y. P. Lu,${ }^{1,47}$ C. L. Luo, ${ }^{34}$ M. X. Luo, ${ }^{66}$ P. W. Luo, ${ }^{48}$ T. Luo, ${ }^{9, j}$ X. L. Luo, ${ }^{1,47}$ S. Lusso,${ }^{62 c}$ X. R. Lyu ${ }^{51}$ F. C. Ma ${ }^{33}$ H. L. Ma, ${ }^{1}$ L. L. Ma, ${ }^{40}$ M. M. Ma,${ }^{1,51}$ Q. M. Ma, ${ }^{1}$

R. Q. Ma ${ }^{1,51}$ R. T. Ma ${ }^{51}$ X. N. Ma ${ }^{36}$ X. X. Ma,${ }^{1,51}$ X. Y. Ma, ${ }^{1,47}$ Y. M. Ma, ${ }^{40}$ F. E. Maas, ${ }^{15}$ M. Maggiora, ${ }^{62 a, 62 c}$

S. Maldaner, ${ }^{27}$ S. Malde,${ }^{57}$ Q. A. Malik, ${ }^{61}$ A. Mangoni, ${ }^{23 b}$ Y. J. Mao, ${ }^{37, m}$ Z. P. Mao, ${ }^{1}$ S. Marcello, ${ }^{62,62 c}$ Z. X. Meng, ${ }^{53}$ J. G. Messchendorp, ${ }^{30}$ G. Mezzadri, ${ }^{24 a}$ J. Min, ${ }^{1,47}$ T. J. Min, ${ }^{35}$ R. E. Mitchell, ${ }^{22}$ X. H. Mo, ${ }^{1,47,51}$ Y. J. Mo, ${ }^{6}$

C. Morales Morales, ${ }^{15}$ N. Yu. Muchnoi, ${ }^{10, \mathrm{e}}$ H. Muramatsu, ${ }^{55}$ S. Nakhoul,${ }^{11, h}$ Y. Nefedov, ${ }^{28}$ F. Nerling, ${ }^{11, h}$ I. B. Nikolaev,,${ }^{10, e}$ Z. Ning, ${ }^{1,47}$ S. Nisar, ${ }^{8, k}$ S. L. Olsen, ${ }^{51}$ Q. Ouyang, ${ }^{1,47,51}$ S. Pacetti, ${ }^{23 b}$ X. Pan, ${ }^{45}$ Y. Pan, ${ }^{54}$ M. Papenbrock, ${ }^{63}$ A. Pathak, ${ }^{1}$ P. Patteri, ${ }^{23 a}$ M. Pelizaeus, ${ }^{4}$ H. P. Peng,${ }^{59,47}$ K. Peters,,${ }^{11, h}$ J. Pettersson,${ }^{63}$ J. L. Ping, ${ }^{34}$ R. G. Ping,${ }^{1,51}$ A. Pitka, ${ }^{4}$ R. Poling,${ }^{55}$ V. Prasad,${ }^{59,47}$ H. Qi,${ }^{59,47}$ H. R. Qi ${ }^{49}$ M. Qi,${ }^{35}$ T. Y. Qi, ${ }^{2}$ S. Qian, ${ }^{1,47}$ C. F. Qiao, ${ }^{51}$ L. Q. Qin, ${ }^{12}$ X. P. Qin, ${ }^{13}$ X. S. Qin, ${ }^{4}$ Z. H. Qin, ${ }^{1,47}$ J. F. Qiu, ${ }^{1}$ S. Q. Qu ${ }^{36}$ K. H. Rashid ${ }^{61}$ K. Ravindran, ${ }^{21}$ C. F. Redmer, ${ }^{27}$ M. Richter, ${ }^{4}$ A. Rivetti, ${ }^{62 c}$ V. Rodin,${ }^{30}$

M. Rolo, ${ }^{62 c}$ G. Rong, ${ }^{1,51}$ Ch. Rosner, ${ }^{15}$ M. Rump ${ }^{56}$ A. Sarantsev, ${ }^{28, f}$ M. Savrié, ${ }^{24 b}$ Y. Schelhaas, ${ }^{27}$ C. Schnier, ${ }^{4}$

K. Schoenning, ${ }^{63}$ D. C. Shan, ${ }^{45}$ W. Shan, ${ }^{19}$ X. Y. Shan, ${ }^{59,47}$ M. Shao, ${ }^{59,47}$ C. P. Shen, ${ }^{2}$ P. X. Shen, ${ }^{36}$ X. Y. Shen, ${ }^{1,51}$ H. Y. Sheng, ${ }^{1}$ H. C. Shi,${ }^{59,47}$ R. S. Shi,${ }^{1,51}$ X. Shi, ${ }^{1,47}$ X. D. Shi, ${ }^{59,47}$ J. J. Song ${ }^{40}$ Q. Q. Song, ${ }^{59,47}$ X. Y. Song, ${ }^{1}$ Y. X. Song, ${ }^{37, m}$ S. Sosio, ${ }^{62 a, 62 c}$ C. Sowa, ${ }^{4}$ S. Spataro, ${ }^{62 a, 62 c}$ F. F. Sui, ${ }^{40}$ G. X. Sun, ${ }^{1}$ J. F. Sun, ${ }^{16}$ L. Sun, ${ }^{64}$ S. S. Sun, ${ }^{1,51}$ T. Sun, ${ }^{1,51}$ W. Y. Sun,${ }^{34}$ Y. J. Sun, ${ }^{59,47}$ Y. K. Sun, ${ }^{59,47}$ Y.Z. Sun, ${ }^{1}$ Z. J. Sun, ${ }^{1,47}$ Z. T. Sun, ${ }^{1}$ Y. X. Tan,${ }^{59,47}$ C. J. Tang, ${ }^{44}$ G. Y. Tang, ${ }^{1}$ J. Tang, ${ }^{48}$

X. Tang, ${ }^{1}$ V. Thoren,${ }^{63}$ B. Tsednee, ${ }^{26}$ I. Uman, ${ }^{50 \mathrm{~d}}$ B. Wang, ${ }^{1}$ B. L. Wang, ${ }^{51}$ C. W. Wang, ${ }^{35}$ D. Y. Wang, ${ }^{37, m}$ H. P. Wang,,${ }^{1,51}$ K. Wang, ${ }^{1,47}$ L. L. Wang, ${ }^{1}$ L. S. Wang, ${ }^{1}$ M. Wang, ${ }^{40}$ M. Z. Wang, ${ }^{37, m}$ Meng Wang, ${ }^{1,51}$ P. L. Wang, ${ }^{1}$ W. P. Wang, ${ }^{59,47}$

X. Wang, ${ }^{37, \mathrm{~m}}$ X. F. Wang, ${ }^{31}$ X. L. Wang, ${ }^{9, j}$ Y. Wang, ${ }^{59,47}$ Y. Wang, ${ }^{48}$ Y. D. Wang, ${ }^{15}$ Y. F. Wang, ${ }^{1,47,51}$ Y. Q. Wang, ${ }^{1}$

Z. Wang, ${ }^{1,47}$ Z. G. Wang, ${ }^{1,47}$ Z. Y. Wang, ${ }^{1}$ Ziyi Wang, ${ }^{51}$ Zongyuan Wang, ${ }^{1,51}$ T. Weber, ${ }^{4}$ D. H. Wei, ${ }^{12}$ P. Weidenkaff, ${ }^{27}$

F. Weidner, ${ }^{56}$ H. W. Wen, ${ }^{34, a}$ S. P. Wen, ${ }^{1}$ D. J. White, ${ }^{54}$ U. Wiedner, ${ }^{4}$ G. Wilkinson, ${ }^{57}$ M. Wolke, ${ }^{63}$ L. Wollenberg, ${ }^{4}$

J. F. Wu, ${ }^{1,51}$ L. H. Wu, ${ }^{1}$ L. J. Wu, ${ }^{1,51}$ X. Wu,${ }^{9, j}$ Z. Wu,,${ }^{1,47}$ L. Xia, ${ }^{59,47}$ H. Xiao, ${ }^{9, j}$ S. Y. Xiao, ${ }^{1}$ Y. J. Xiao, ${ }^{1,51}$ Z. J. Xiao, ${ }^{34}$ Y. G. Xie, ${ }^{1,47}$ Y. H. Xie, ${ }^{6}$ T. Y. Xing, ${ }^{1,51}$ X. A. Xiong, ${ }^{1,51}$ G. F. Xu, ${ }^{1}$ J. J. Xu, ${ }^{35}$ Q. J. Xu, ${ }^{14}$ W. Xu, ${ }^{1,51}$ X. P. Xu,,${ }^{45}$ L. Yan, ${ }^{62 a, 62 c}$ L. Yan, ${ }^{9, j}$ W. B. Yan,${ }^{59,47}$ W. C. Yan, ${ }^{67}$ Xu Yan, ${ }^{45}$ H. J. Yang, ${ }^{41, i}$ H. X. Yang, ${ }^{1}$ L. Yang, ${ }^{64}$ R. X. Yang, ${ }^{59,47}$ S. L. Yang,,${ }^{1,51}$ Y. H. Yang, ${ }^{35}$ Y. X. Yang, ${ }^{12}$ Yifan Yang, ${ }^{1,51}$ Zhi Yang, ${ }^{25}$ M. Ye, ${ }^{1,47}$ M. H. Ye, ${ }^{7}$ J. H. Yin, ${ }^{1}$ Z. Y. You, ${ }^{48}$ B. X. Yu, ${ }^{1,47,51}$ 
C. X. Yu, ${ }^{36}$ G. Yu, ${ }^{1,51}$ J. S. Yu, ${ }^{20, n}$ T. Yu, ${ }^{60}$ C. Z. Yuan, ${ }^{1,51}$ W. Yuan, ${ }^{62 a, 62 c}$ X. Q. Yuan, ${ }^{37, m}$ Y. Yuan, ${ }^{1}$ C. X. Yue, ${ }^{32}$ A. Yuncu, ${ }^{50 b, b}$ A. A. Zafar, ${ }^{61}$ Y. Zeng, ${ }^{20, n}$ B. X. Zhang, ${ }^{1}$ B. Y. Zhang, ${ }^{1,47}$ C. C. Zhang, ${ }^{1}$ D. H. Zhang, ${ }^{1}$ Guangyi Zhang, ${ }^{16}$ H. H. Zhang, ${ }^{48}$ H. Y. Zhang, ${ }^{1,47}$ J. L. Zhang, ${ }^{65}$ J. Q. Zhang, ${ }^{4}$ J. W. Zhang, ${ }^{1,47,51}$ J. Y. Zhang, ${ }^{1}$ J. Z. Zhang, ${ }^{1,51}$ Jianyu Zhang, ${ }^{1,51}$ Jiawei Zhang, ${ }^{1,51}$ L. Zhang, ${ }^{1}$ Lei Zhang, ${ }^{35}$ S. Zhang, ${ }^{48}$ S. F. Zhang, ${ }^{35}$ T. J. Zhang, ${ }^{41, i}$ X. Y. Zhang, ${ }^{40}$ Y. Zhang, ${ }^{57}$ Y. H. Zhang, ${ }^{1,47}$ Y. T. Zhang $\odot,{ }^{59,47,{ }^{*}}$ Yan Zhang, ${ }^{59,47}$ Yao Zhang, ${ }^{1}$ Yi Zhang, ${ }^{9, j}$ Z. H. Zhang, ${ }^{6}$ Z. Y. Zhang, ${ }^{64}$ G. Zhao, ${ }^{1}$ J. Zhao, ${ }^{32}$ J. W. Zhao, ${ }^{1,47}$ J. Y. Zhao, ${ }^{1,51}$ J. Z. Zhao, ${ }^{1,47}$ Lei Zhao ${ }^{59,47}$ Ling Zhao, ${ }^{1}$ M. G. Zhao, ${ }^{36}$ Q. Zhao, ${ }^{1}$ S. J. Zhao, ${ }^{67}$ T. C. Zhao, ${ }^{1}$ Y. B. Zhao, ${ }^{1,47}$ Z. G. Zhao, ${ }^{59,47}$ A. Zhemchugov, ${ }^{28, c}$ B. Zheng, ${ }^{60}$ J. P. Zheng, ${ }^{1,47}$ Y. Zheng, ${ }^{37, m}$ Y. H. Zheng, ${ }^{51}$ B. Zhong, ${ }^{34}$ C. Zhong, ${ }^{60}$ L. Zhou, ${ }^{1,47}$ L. P. Zhou, ${ }^{1,51}$ Q. Zhou, ${ }^{1,51}$ X. Zhou, ${ }^{64}$ X. K. Zhou, ${ }^{51}$ X. R. Zhou, ${ }^{59,47}$ A. N. Zhu, ${ }^{1,51}$ J. Zhu, ${ }^{36}$ K. Zhu, ${ }^{1}$ K. J. Zhu, ${ }^{1,47,51}$ S. H. Zhu, ${ }^{58}$ W. J. Zhu, ${ }^{36}$ X. L. Zhu, ${ }^{49}$ Y. C. Zhu, ${ }^{59,47}$ Y. S. Zhu, ${ }^{1,51}$ Z. A. Zhu, ${ }^{1,51}$ J. Zhuang, ${ }^{1,47}$ B. S. Zou, ${ }^{1}$ and J. H. Zou ${ }^{1}$

\title{
(BESIII Collaboration)
}

\author{
${ }^{1}$ Institute of High Energy Physics, Beijing 100049, People's Republic of China \\ ${ }^{2}$ Beihang University, Beijing 100191, People's Republic of China \\ ${ }^{3}$ Beijing Institute of Petrochemical Technology, Beijing 102617, People's Republic of China \\ ${ }^{4}$ Bochum Ruhr-University, D-44780 Bochum, Germany \\ ${ }^{5}$ Carnegie Mellon University, Pittsburgh, Pennsylvania 15213, USA \\ ${ }^{6}$ Central China Normal University, Wuhan 430079, People's Republic of China \\ ${ }^{7}$ China Center of Advanced Science and Technology, Beijing 100190, People's Republic of China \\ ${ }^{8}$ COMSATS University Islamabad, Lahore Campus, Defence Road, Off Raiwind Road, 54000 Lahore, Pakistan \\ ${ }^{9}$ Fudan University, Shanghai 200443, People's Republic of China \\ ${ }^{10}$ G.I. Budker Institute of Nuclear Physics SB RAS (BINP), Novosibirsk 630090, Russia \\ ${ }^{11}$ GSI Helmholtzcentre for Heavy Ion Research GmbH, D-64291 Darmstadt, Germany \\ ${ }^{12}$ Guangxi Normal University, Guilin 541004, People's Republic of China \\ ${ }^{13}$ Guangxi University, Nanning 530004, People's Republic of China \\ ${ }^{14}$ Hangzhou Normal University, Hangzhou 310036, People's Republic of China \\ ${ }^{15}$ Helmholtz Institute Mainz, Johann-Joachim-Becher-Weg 45, D-55099 Mainz, Germany \\ ${ }^{16}$ Henan Normal University, Xinxiang 453007, People's Republic of China \\ ${ }^{17}$ Henan University of Science and Technology, Luoyang 471003, People's Republic of China \\ ${ }^{18}$ Huangshan College, Huangshan 245000, People's Republic of China \\ ${ }^{19}$ Hunan Normal University, Changsha 410081, People's Republic of China \\ ${ }^{20}$ Hunan University, Changsha 410082, People's Republic of China \\ ${ }^{21}$ Indian Institute of Technology Madras, Chennai 600036, India \\ ${ }^{22}$ Indiana University, Bloomington, Indiana 47405, USA \\ ${ }^{23 a}$ INFN Laboratori Nazionali di Frascati, I-00044 Frascati, Italy \\ ${ }^{23 \mathrm{~b}}$ INFN and University of Perugia, I-06100 Perugia, Italy \\ ${ }^{24 \mathrm{a}}$ INFN Sezione di Ferrara, I-44122 Ferrara, Italy \\ ${ }^{24 \mathrm{~b}}$ University of Ferrara, I-44122 Ferrara, Italy \\ ${ }^{25}$ Institute of Modern Physics, Lanzhou 730000, People's Republic of China \\ ${ }^{26}$ Institute of Physics and Technology, Peace Avenue 54B, Ulaanbaatar 13330, Mongolia \\ ${ }^{27}$ Johannes Gutenberg University of Mainz, Johann-Joachim-Becher-Weg 45, D-55099 Mainz, Germany \\ ${ }^{28}$ Joint Institute for Nuclear Research, Dubna 141980, Moscow region, Russia \\ ${ }^{29}$ Justus-Liebig-Universitaet Giessen, II. Physikalisches Institut, Heinrich-Buff-Ring 16, D-35392 Giessen, Germany \\ ${ }^{30}$ KVI-CART, University of Groningen, NL-9747 AA Groningen, Netherlands \\ ${ }^{31}$ Lanzhou University, Lanzhou 730000, People's Republic of China \\ ${ }^{32}$ Liaoning Normal University, Dalian 116029, People's Republic of China \\ ${ }^{33}$ Liaoning University, Shenyang 110036, People's Republic of China \\ ${ }^{34}$ Nanjing Normal University, Nanjing 210023, People's Republic of China \\ ${ }^{35}$ Nanjing University, Nanjing 210093, People's Republic of China \\ ${ }^{36}$ Nankai University, Tianjin 300071, People's Republic of China \\ ${ }^{37}$ Peking University, Beijing 100871, People's Republic of China \\ ${ }^{38}$ Qufu Normal University, Qufu 273165, People's Republic of China \\ ${ }^{39}$ Shandong Normal University, Jinan 250014, People's Republic of China \\ ${ }^{40}$ Shandong University, Jinan 250100, People's Republic of China \\ ${ }^{41}$ Shanghai Jiao Tong University, Shanghai 200240, People's Republic of China \\ ${ }^{42}$ Shanxi Normal University, Linfen 041004, People's Republic of China \\ ${ }^{43}$ Shanxi University, Taiyuan 030006, People's Republic of China
}




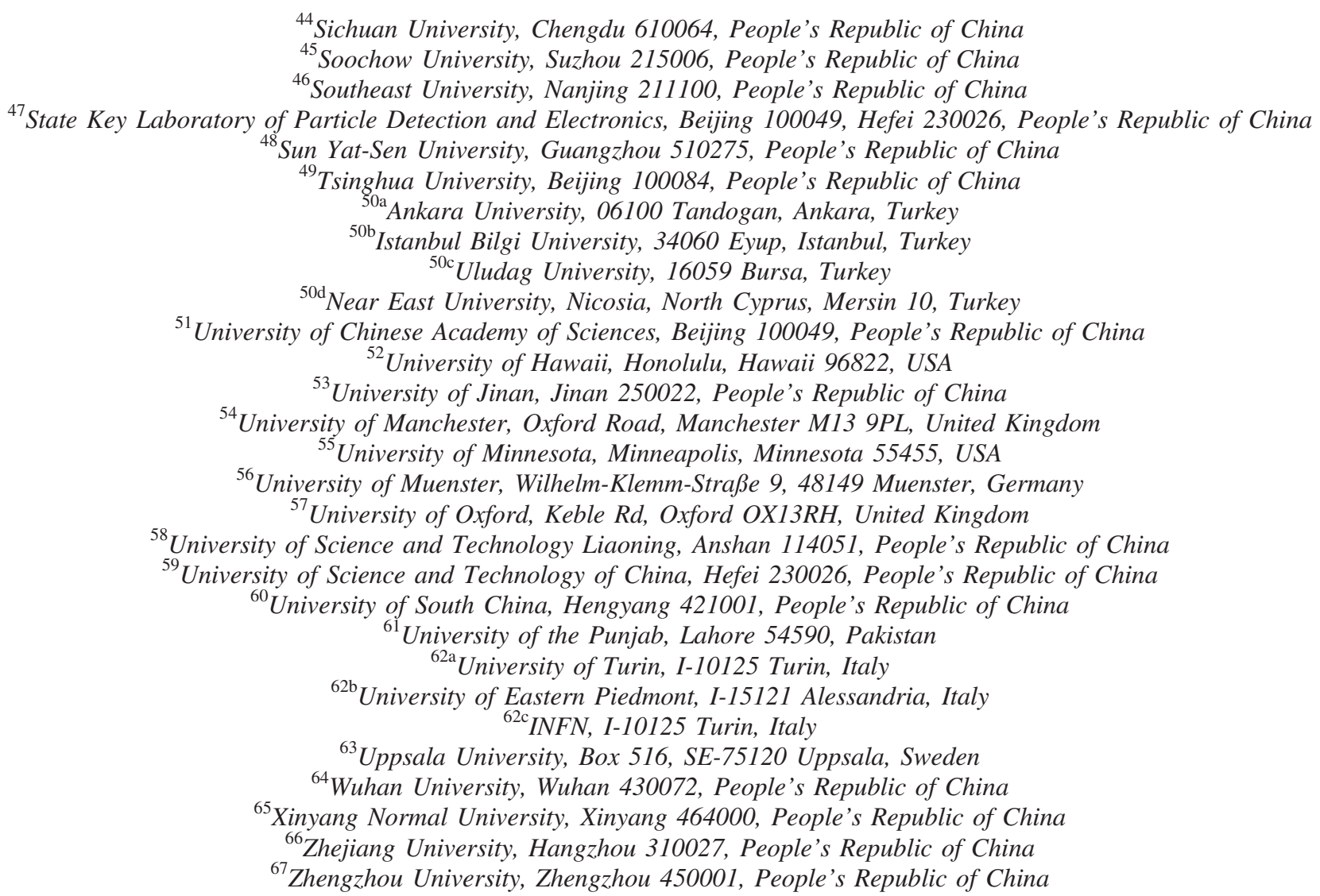

(Received 14 January 2020; accepted 28 February 2020; published 19 March 2020)

A partial-wave analysis is performed for the process $e^{+} e^{-} \rightarrow K^{+} K^{-} \pi^{0} \pi^{0}$ at the center-of-mass energies ranging from 2.000 to $2.644 \mathrm{GeV}$. The data samples of $e^{+} e^{-}$collisions, collected by the BESIII detector at the BEPCII collider with a total integrated luminosity of $300 \mathrm{pb}^{-1}$, are analyzed. The total Born cross sections for the process $e^{+} e^{-} \rightarrow K^{+} K^{-} \pi^{0} \pi^{0}$, as well as the Born cross sections for the subprocesses $e^{+} e^{-} \rightarrow \phi \pi^{0} \pi^{0}, K^{+}(1460) K^{-}, K_{1}^{+}(1400) K^{-}, K_{1}^{+}(1270) K^{-}$, and $K^{*+}(892) K^{*-}(892)$, are measured versus the center-of-mass energy. The corresponding results for $e^{+} e^{-} \rightarrow K^{+} K^{-} \pi^{0} \pi^{0}$ and $\phi \pi^{0} \pi^{0}$ are consistent with those of $B A B A R$ with better precision. By analyzing the cross sections for the four subprocesses, $K^{+}(1460) K^{-}, K_{1}^{+}(1400) K^{-}, K_{1}^{+}(1270) K^{-}$, and $K^{*+}(892) K^{*-}(892)$, a structure with mass $M=$ $(2126.5 \pm 16.8 \pm 12.4) \mathrm{MeV} / c^{2}$ and width $\Gamma=(106.9 \pm 32.1 \pm 28.1) \mathrm{MeV}$ is observed with an overall statistical significance of $6.3 \sigma$, although with very limited significance in the subprocesses $e^{+} e^{-} \rightarrow$ $K_{1}^{+}(1270) K^{-}$and $K^{*+}(892) K^{*-}(892)$. The resonant parameters of the observed structure suggest it can be identified with the $\phi(2170)$, thus the results provide valuable input to the internal nature of the $\phi(2170)$.

DOI: 10.1103/PhysRevLett.124.112001

The vector meson state $Y(2175)$, denoted as the $\phi(2170)$ by the Particle Data Group (PDG) [1], is currently one of the most interesting particles in light hadron spectroscopy. The $\phi(2170)$ was first observed by $B A B A R$ [2] and subsequently

Published by the American Physical Society under the terms of the Creative Commons Attribution 4.0 International license. Further distribution of this work must maintain attribution to the author(s) and the published article's title, journal citation, and DOI. Funded by SCOAP . confirmed by several other experiments [3-8]. The internal constituents of the $\phi(2170)$ are still unknown, which has stimulated extensive theoretical discussions. Possible interpretations of the $\phi(2170)$ include a conventional $3^{3} S_{1}$ or $2^{3} D_{1} s \bar{s}$ state [9-12], an $s \bar{s} g$ hybrid [10,13,14], a tetraquark state [15-18], a $\Lambda \bar{\Lambda}\left({ }^{3} S_{1}\right)$ bound state [19-21], or a $\phi K K$ resonance state [22], etc., but no interpretation has yet been established. Each of these theoretical models can accommodate a resonant state with parameters similar to those of the $\phi(2170)$, but they predict significantly different partial 
widths for individual decay modes, especially the $K^{(*)} K^{(*)}$ decay modes, where the $K^{(*)}$ is the ground or excited state of a $K$ meson with different spin parities. Consequently, studying the decay modes of the $\phi(2170)$, and precisely measuring their partial widths, plays a key role in determining the internal structure of the $\phi(2170)$.

The BESII Collaboration searched for the decay $\phi(2170) \rightarrow K^{* 0}(892) \bar{K}^{* 0}(892)$ via $J / \psi \rightarrow \eta \phi(2170)$ by using 58 million $J / \psi$ events [23]. No significant signal was observed. The BABAR Collaboration performed an analysis of $e^{+} e^{-} \rightarrow K^{+} K^{-} \pi^{+} \pi^{-}$and $K^{+} K^{-} \pi^{0} \pi^{0}$ using $454 \mathrm{fb}^{-1}$ data via the initial state radiation (ISR) process [4]. Beside clearly observing the process $e^{+} e^{-} \rightarrow \phi \pi \pi$, abundant $K^{*}$ structures were observed in the $K \pi(\pi)$ invariant mass spectrum, such as the $K^{*}(892)$ and $K_{2}^{*}(1430)$, as well as the $K_{1}(1270)$ and $K_{1}(1400)$. It is worth noting that only about $1 \%$ of the $e^{+} e^{-} \rightarrow K^{+} K^{-} \pi^{+} \pi^{-}$events were from the subprocess $e^{+} e^{-} \rightarrow K^{* 0}(892) \bar{K}^{* 0}(892)$, while roughly $30 \%$ of the $e^{+} e^{-} \rightarrow K^{+} K^{-} \pi^{0} \pi^{0}$ events were from $e^{+} e^{-} \rightarrow K^{*+}(892) K^{*-}(892)$. A comprehensive analysis, e.g., a partial-wave analysis (PWA), is desired to resolve the contribution of individual components in these decays.

Besides an excited $\phi$ state, the quark model also predicts excited $\rho$ and $\omega$ states in the $2 \mathrm{GeV} / c^{2}$ mass range [24]. Finding this set of excited vector mesons would help establish the corresponding $\rho, \omega$, and $\phi$ meson families and would set a baseline for theoretical models. Since these excited vector mesons can each decay into $K^{(*)} K^{(*)}$ final states, analyzing the $K^{(*)} K^{(*)}$ invariant mass spectra in $e^{+} e^{-}$ annihilation becomes an effective means to discover them.

In this Letter, we present a PWA of the process $e^{+} e^{-} \rightarrow$ $K^{+} K^{-} \pi^{0} \pi^{0}$ using data collected with the BESIII detector. The ten data samples used in this analysis have center-ofmass (c.m.) energies ranging from 2.000 to $2.644 \mathrm{GeV}$ and have a total integrated luminosity of $300 \mathrm{pb}^{-1}$. The c.m. energy values and integrated luminosities of each dataset are presented in Table I in the Supplemental Material [25]. Charge-conjugated processes are always included by default.

Detailed descriptions of the design and performance of the BESIII detector can be found in Ref. [26]. A Monte Carlo (MC) simulation based on GEANT4 [27], including the geometric description of the BESIII detector and its response, is used to optimize the event selection criteria, estimate backgrounds, and determine the detection efficiency. The signal MC samples are generated using the package CONEXC [28], which incorporates a higher-order ISR correction. Background samples of the processes $e^{+} e^{-} \rightarrow e^{+} e^{-}, \mu^{+} \mu^{-}$, and $\gamma \gamma$ are generated with the BABAYAGA [29] generator, while $e^{+} e^{-} \rightarrow$ hadrons and two photon events are generated by the LUARLW [30] and BESTWOGAM [31] generators, respectively.

The selection criteria for charged tracks, particle identification (PID), and photon candidates are the same as those in Ref. [32].
The process $e^{+} e^{-} \rightarrow K^{+} K^{-} \pi^{0} \pi^{0}$ results in the final state $K^{+} K^{-} \gamma \gamma \gamma \gamma$. Thus, candidate events with only two oppositely charged kaons and at least four photons are selected. To improve the kinematic resolution and suppress background, a six-constraint (6C) kinematic fit imposing energy-momentum conservation, as well as two additional $\pi^{0}$ mass constraints, is carried out under the hypothesis $e^{+} e^{-} \rightarrow K^{+} K^{-} \pi^{0} \pi^{0}$. The combination with minimum $\chi_{6 C}^{2}$ is retained for further analysis. The candidate events are required to satisfy $\chi_{6 C}^{2}<80$. After the above selection criteria, detailed studies indicate that the backgrounds are negligible.

Using the GPUPWA framework [33], a PWA is performed on the surviving candidate events to disentangle the intermediate processes present in $e^{+} e^{-} \rightarrow K^{+} K^{-} \pi^{0} \pi^{0}$. The quasi two-body decay amplitudes in the sequential decays are constructed using covariant tensor amplitudes [34]. The intermediate states are parametrized with relativistic BreitWigner (BW) functions, except for the $f_{0}(980)$, which is described with a Flatté formula [35]. The resonance parameters of the $f_{0}(980)$ and the wide resonance $\sigma$ in the fit are fixed to those in Ref. [35] and Refs. [35,36], respectively, and those of other intermediate states are fixed to PDG values, or measured in the analysis. To include the resolution for the narrow $\phi(1020)$ resonance, a Gaussian function is convolved with the BW function, but this is not done for the other resonances. The relative magnitudes and phases of the individual intermediate processes are determined by performing an unbinned maximum likelihood fit using MINUIT [37].

We start the fit procedure by including all possible intermediate states in the PDG that conserve $\mathrm{J}^{\mathrm{PC}}$, where these intermediate states can decay into $K^{+} K^{-}, \pi^{0} \pi^{0}$, $K^{ \pm} \pi^{0}, K^{+} K^{-} \pi^{0}$, or $K^{ \pm} \pi^{0} \pi^{0}$ final states. Then we examine the statistical significance of the individual amplitudes, and drop the ones with statistical significance less than $5 \sigma$. The process is repeated until no amplitude remains with a statistical significance less than $5 \sigma$. After that, all the removed processes are reintroduced individually to make sure that they are not needed in the fit. In the above approach, the statistical significance of each individual amplitude is determined by the changes in the negative log likelihood (NLL) value and the number of free parameters in the fit with and without the corresponding amplitude included.

The above strategy is performed individually on the datasets at $\sqrt{s}=2.125$ and $2.396 \mathrm{GeV}$, which have the largest luminosities among the ten datasets. The nominal solution for data at $\sqrt{s}=2.125 \mathrm{GeV}$ includes the twobody decay processes $K^{+}(1460) K^{-}, \quad K_{1}^{+}(1270) K^{-}$, $K_{1}^{+}(1400) K^{-}, K^{*+}(892) K^{*-}(892), K_{0}^{*+}(1430) K^{*-}(892)$, $\phi(1020) \sigma, \quad \phi(1020) f_{0}(980), \quad \phi(1020) f_{2}(1270), \quad$ and $\omega(1420) \pi^{0}$, as well as the three-body decay processes $K^{+} K^{-} \sigma, K^{+} K^{-} f_{0}(980)$, and $K^{+} K^{-} f_{0}(1370)$. For the 
data at $\sqrt{s}=2.396 \mathrm{GeV}$, the additional intermediate processes $K_{2}^{*+}(1430) K^{*-}(892), \quad K^{*+}(892) K^{-} \pi^{0}$, and $\phi(1020) f_{0}(1370)$ are included, but without the $\phi(1020) \sigma$ and $\phi(1020) f_{2}(1270)$ processes. An interesting decay mode $K^{*+}(1410) K^{-}$, which is expected to have a sizable decay rate for a conventional $3^{3} S_{1} s \bar{s}$ state [10], is found to be less than $3 \sigma$ in both data samples. In the above, the three-body decays are treated as consecutive quasi twobody decays with a very broad resonance decaying into $K^{+} K^{-}$or $K^{+} \pi^{0}$ and modeled as a $1^{-}$phase space distribution. The intermediate states $K^{+}(1460), K_{1}^{+}(1270)$, $K_{1}^{+}(1400)$ decay into $K^{*+}(892) \pi^{0}$, and $\omega(1420)$ decays into $K^{* \pm}(892) K^{\mp}$, followed by $K^{*+}(892) \rightarrow K^{+} \pi^{0}$. The state $K_{0}^{*+}(1430)$ decays into $K^{+} \pi^{0}$. The state $\phi(1020)$ decays into $K^{+} K^{-}$and $\sigma, f_{0}(980), f_{2}(1270), f_{0}(1370)$ decay into $\pi^{0} \pi^{0}$. The masses and widths of the $K(1460)$, $K_{1}(1400), K_{1}(1270)$, and $\omega(1420)$ in the fit are determined by scanning the likelihood value, and the results are consistent with the parameters in the PDG. The masses and widths of other intermediate states are fixed to PDG values. The statistical significance of all intermediate processes are summarized in Secs. II and III of the Supplemental Material [25], respectively. The corresponding comparison of invariant mass spectra and angular distributions between data and the MC projections are shown in Sec. IV of the Supplemental Material.

For the other eight data samples, due to limited statistics, we do not perform the above optimization strategy to determine which intermediate processes to include. Instead, we use the same intermediate processes as the datasets with nearby c.m. energy. The datasets with $\sqrt{s}=2.000,2.100$, $2.175,2.200$, and $2.232 \mathrm{GeV}$ (referred to as group I data), use the same processes as $\sqrt{s}=2.125 \mathrm{GeV}$, while the other three points (group II data) use the same processes as $\sqrt{s}=2.396 \mathrm{GeV}$.

The total Born cross sections for $e^{+} e^{-} \rightarrow K^{+} K^{-} \pi^{0} \pi^{0}$ and the Born cross sections for the intermediate processes are obtained at each c.m. energy using

$$
\sigma^{B}=\frac{N^{\text {sig }}}{\mathcal{L}_{\text {int }} \frac{1}{|1-\Pi|^{2}}(1+\delta)^{r} \mathcal{B} r \epsilon},
$$

where $N^{\text {sig }}$ is the corresponding signal yield, and is determined by calculating the fraction according to the PWA results for the individual intermediate process; $\mathcal{L}_{\text {int }}$ is the integrated luminosity; $(1+\delta)^{r}$ is the ISR correction factor obtained from a QED calculation [28,38] and incorporating the input cross section in this analysis, where three iterations are performed until the measured Born cross section does not change by more than $1.0 \%$; $1 /\left(|1-\Pi|^{2}\right)$ is the vacuum polarization factor taken from a QED calculation [39]; $\epsilon$ is the detection efficiency obtained from a PWA-weighted MC sample; and $\mathcal{B} r$ is the product of branching ratios of the intermediate states as quoted in the
PDG [1]. In the decay $e^{+} e^{-} \rightarrow K^{+}(1460) K^{-}$, the branching fraction of $K(1460) \rightarrow K^{*}(892) \pi$ is included in the measured cross section since it has never been measured.

Two categories of systematic uncertainties are considered in the measurement of the Born cross sections. The first category includes uncertainties associated with the luminosity, track detection, PID, kinematic fit, ISR correction, and the branching fractions of intermediate states. The uncertainty associated with the integrated luminosity is $1 \%$ at each energy point [40]. The uncertainty of the detection efficiency is $1 \%$ for each charged track [41] and photon [42]. The PID efficiency uncertainty is $1.0 \%$ for each charged track [41]. The uncertainty related to the kinematic fit is estimated by correcting the helix parameters of the simulated charged tracks to match the resolution [43]. The uncertainty associated with the ISR correction factor is estimated to be the difference of $\left(1+\delta^{r}\right) \epsilon$ between the last two iterations in the cross section measurement. The systematic uncertainties from the branching ratios of intermediate states in the subsequent decays are taken from the PDG [1]. The second category of uncertainties are from the PWA fit procedure. Fits with alternative scenarios are performed, and the changes of signal yields are taken as systematic uncertainties. Uncertainties from the BW parametrization are estimated by replacing the constant-width BW with the mass-dependent width. Uncertainties associated with the resonance parameters, which are taken from the PDG and fixed in the fit, are estimated by alternative fits imposing additional constraints on these resonance parameters. Meanwhile, the imposed constraints follow Gaussian distributions with widths equal to their uncertainties. One thousand fits are performed, and the resultant standard deviations of the signal yields are taken as systematic uncertainties. Uncertainties associated with the additional resonances are estimated by alternative fits including the components $K^{*}(1410) K$ or the $K_{2}^{*}(1430) K^{*}$, which are most significant, but less than $5 \sigma$. Uncertainties due to the barrier factor $[34,44]$ are estimated by varying the radius of the centrifugal barrier from 0.7 to $1.0 \mathrm{fm}$. To estimate the uncertainties on the detection efficiency related to the fit parameters in the PWA, $500 \mathrm{MC}$ samples are generated with 500 groups of parameters of PWA amplitudes, which is sampled from a multivariable Gaussian function according to their mean values and their covariance error matrix from the nominal fit. The standard deviations of the resultant detection efficiencies are considered as the uncertainties.

In the above procedure, the uncertainties associated with the barrier factor, resonance parametrization, and additional resonances are strongly affected by the statistics. Thus, those uncertainties of data with $\sqrt{s}=2.125 \mathrm{GeV}$ are assigned to the group I data, while those of data with $\sqrt{s}=$ $2.396 \mathrm{GeV}$ are assigned to the group II data. Assuming all sources of systematic uncertainties are independent, the total uncertainties are the quadratic sums of the individual 

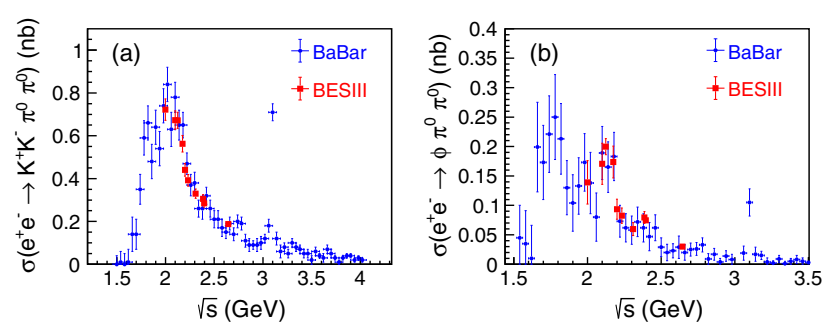

FIG. 1. The Born cross sections for (a) the process $e^{+} e^{-} \rightarrow$ $K^{+} K^{-} \pi^{0} \pi^{0}$ and (b) the subprocess $e^{+} e^{-} \rightarrow \phi \pi^{0} \pi^{0}$. The red squares are from this analysis; the blue dots are from the BABAR experiment [4]. For our data, the errors reflect both statistical and systematical uncertainties.

values, shown in Sec. V of the Supplemental Material [25], where the sources of the uncertainties tagged with an asterisk are assumed to be $100 \%$ correlated among each energy points.

The measured total Born cross sections for $e^{+} e^{-} \rightarrow$ $K^{+} K^{-} \pi^{0} \pi^{0}$ and the Born cross sections for the subprocess $e^{+} e^{-} \rightarrow \phi \pi^{0} \pi^{0}$, summing over all the $\pi^{0} \pi^{0}$ intermediate processes and their interferences, are shown in Fig. 1. Good agreement is found with the previous results from $B A B A R$. In order to study the properties of $1^{--}$states, the cross sections for the processes $e^{+} e^{-} \rightarrow K^{+}(1460) K^{-}$, $K_{1}^{+}(1400) K^{-}, \quad K_{1}^{+}(1270) K^{-}$, and $K^{*+}(892) K^{*-}(892)$, referred to as the $K K$ processes, are shown in Fig. 2. A clear peak between 2.1 and $2.2 \mathrm{GeV}$ is present in the process $e^{+} e^{-} \rightarrow K^{+}(1460) K^{-}$, and dips are observed for
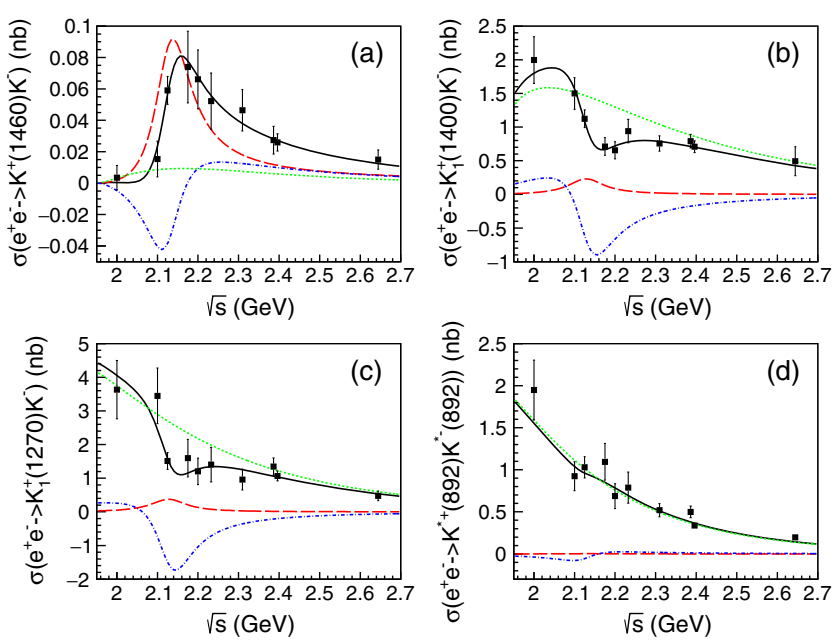

FIG. 2. Fit to the cross sections for $e^{+} e^{-}$to the final states (a) $K^{+}(1460) K^{-}$, (b) $K_{1}^{+}(1400) K^{-}$, (c) $K_{1}^{+}(1270) K^{-}$, and (d) $K^{*+}(892) K^{*-}(892)$, where black dots with errors are data, the black solid curves are the overall fit results, the red longdashed curves are from the intermediate state, the green shortdashed curves are from the nonresonant component, and the blue dash-dotted curves are the interference contribution for solution 1. Here, the errors reflect both statistical and systematic uncertainties. the processes $e^{+} e^{-} \rightarrow K_{1}^{+}(1400) K^{-}$and $K_{1}^{+}(1270) K^{-}$in almost the same energy region. This may be due to destructive interference between different components. No obvious structure or dip is present in the process $e^{+} e^{-} \rightarrow K^{*+}(892) K^{*-}(892)$. All the various numbers used in the cross section calculation are summarized in Sec. I of the Supplemental Material [25].

To further examine the structure, a binned $\chi^{2}$ fit, incorporating the correlated and uncorrelated uncertainties among different energy points, is performed to the cross sections for the $K^{+}(1460) K^{-}, K_{1}^{+}(1400) K^{-}, K_{1}^{+}(1270) K^{-}$, and $K^{*+}(892) K^{*-}(892)$ processes. The fit probability density function (PDF) for the individual processes is the coherent sum of a nonresonant component $f_{1}$ and a resonant component $f_{2}$ :

$$
\mathcal{A}=f_{1}+e^{i \phi} f_{2},
$$

where $\phi$ is the relative phase between the two components. By considering phase space $\Phi(\sqrt{s})$, the energy-dependent cross section of the QED process, and the relative orbital angular momentum $L$ in the two-body decay, the amplitude $f_{1}$ is described as

$$
f_{1}=q^{L} \frac{\sqrt{\Phi(\sqrt{s})}}{s^{n}}
$$

where $q$ is the momentum of the daughter particle, $n$ is a free parameter. The resonant amplitude $f_{2}$ is described with a BW function,

$$
f_{2}=\frac{M_{R}}{\sqrt{s}} \frac{\sqrt{12 \pi \mathcal{B} r \Gamma_{R}^{e^{+} e^{-}} \Gamma_{R}}}{s-M_{R}^{2}+i M_{R} \Gamma_{R}}\left(\frac{q}{q_{0}}\right)^{L} \sqrt{\frac{\Phi(\sqrt{s})}{\Phi\left(M_{R}\right)}},
$$

where $M_{R}$ is the mass of the structure, $\Gamma_{R}$ is the total width, $\Gamma_{R}^{e^{+} e^{-}}$is its partial width to $e^{+} e^{-}, \mathcal{B r}$ is the decay branching fraction to a given final state, and $q_{0}$ is the momenta of the daughter particle in the rest frame of the parent particle $\left(M_{R}\right)$.

A simultaneous fit, assuming the same structure among the $K^{+}(1460) K^{-}, K_{1}^{+}(1400) K^{-}, K_{1}^{+}(1270) K^{-}$, and $K^{*+}(892) K^{*-}(892)$ processes, is performed to the measured cross sections, as shown in Fig. 2. In the fit, $M_{R}$ and $\Gamma_{R}$ are shared parameters between the four processes and are floated, while $n$, the production $\mathcal{B} r \Gamma_{R}^{e^{+} e^{-}}$, and the relative phase angle $\phi$ are floated and final state dependent. For $e^{+} e^{-} \rightarrow K_{1}^{+}(1270) K^{-}$and $K_{1}^{+}(1400) K^{-}, L=0$, while $L=1$ for the other two modes. The fit results have two solutions with equal fit quality, identical $M_{R}=(2126.5 \pm$ 16.8) $\mathrm{MeV} / c^{2}$ and $\Gamma_{R}=(106.9 \pm 32.1) \mathrm{MeV}$, but different $\mathcal{B} r \Gamma_{R}^{e^{+}} e^{-}$and $\phi$ for the processes $e^{+} e^{-} \rightarrow K_{1}^{+}(1400) K^{-}$ and $K_{1}^{+}(1270) K^{-}$, as summarized in Table I. The statistical significance of the structure is estimated with the change of $\chi^{2}\left(\Delta \chi^{2}\right)$ and the number of degrees of freedom ( $\Delta$ ndof) between the scenarios with and without the structure included in the fit. The overall statistical significance is 
TABLE I. A summary of fit results.

\begin{tabular}{lcccc}
\hline \hline Channel & & $\begin{array}{c}\mathcal{B}_{r} \Gamma_{R}^{e^{+} e^{-}} \\
(\mathrm{eV})\end{array}$ & $\begin{array}{c}\phi \\
(\mathrm{rad})\end{array}$ & $\begin{array}{c}\text { signific- } \\
\text { ance }(\sigma)\end{array}$ \\
\hline$K^{+}(1460) K^{-}$ & & $3.0 \pm 3.8$ & $5.6 \pm 1.5$ & 4.4 \\
$K_{1}^{+}(1400) K^{-}$ & Solution 1 & $4.7 \pm 3.3$ & $3.7 \pm 0.4$ & 4.8 \\
& Solution 2 & $98.8 \pm 7.8$ & $4.5 \pm 0.3$ & \\
$K_{1}^{+}(1270) K^{-}$ & Solution 1 & $7.6 \pm 3.7$ & $4.0 \pm 0.2$ & 1.4 \\
& Solution 2 & $152.6 \pm 14.2$ & $4.5 \pm 0.1$ & \\
$K^{*+}(892) K^{*-}(892)$ & & $0.04 \pm 0.2$ & $5.8 \pm 1.9$ & 1.2 \\
\hline \hline
\end{tabular}

$6.3 \sigma$, obtained with $\Delta \chi^{2}=63.8$ and $\Delta$ ndof $=10$. The significance of the resonant state for each $K K$ process is also estimated and summarized in Table I. The significances of the resonant state in the processes $e^{+} e^{-} \rightarrow$ $K^{+}(1460) K^{-}$and $K_{1}^{+}(1400) K^{-}$are greater than $4.5 \sigma$, while no significant signal is found in the other two processes. We also estimate the upper limit at the $90 \%$ confidence level on the production $\mathcal{B} r \Gamma_{R}^{e^{+} e^{-}}$to be $1.9 \mathrm{eV}$ for $e^{+} e^{-} \rightarrow K^{*+}(892) K^{*-}(892)$ and $12.5(297.6) \mathrm{eV}$ for $e^{+} e^{-} \rightarrow K_{1}^{+}(1270) K^{-}$.

The systematic uncertainties on the resonant parameters come from the absolute c.m. energy measurement, the measured cross section, and the fit procedure. The uncertainty of the c.m. energy from BEPCII is small, and is ignored in the determination of the parameters of the structure. The statistical and systematic uncertainties of the measured cross section are incorporated in the fit, thus no further uncertainty is necessary. The uncertainties associated with the fit procedure include those from the fit range and signal model. The uncertainty from the fit range is investigated by excluding the last energy point $\sqrt{s}=$ $2.644 \mathrm{GeV}$ in the fit. The resultant changes, $5.1 \mathrm{MeV} / c^{2}$ for mass and $9.1 \mathrm{MeV}$ for width, are taken as the systematic uncertainties. To assess the systematic uncertainty associated with the signal model, an alternative BW function with energy-dependent width is implemented in the fit, and results in differences of $11.3 \mathrm{MeV} / c^{2}$ and $26.5 \mathrm{MeV}$ for mass and width, respectively, which are taken as the systematic uncertainties. The overall systematic uncertainties are the quadratic sum of the individual ones, $12.4 \mathrm{MeV} / c^{2}$ and $28.1 \mathrm{MeV}$ for the mass and width, respectively.

In summary, a PWA of the process $e^{+} e^{-} \rightarrow K^{+} K^{-} \pi^{0} \pi^{0}$ is performed for ten data samples with c.m. energies from 2.000 to $2.644 \mathrm{GeV}$ and with an integrated luminosity of $300 \mathrm{pb}^{-1}$. The Born cross sections for $e^{+} e^{-} \rightarrow K^{+} K^{-} \pi^{0} \pi^{0}$ and $\phi \pi^{0} \pi^{0}$ are obtained and are consistent with those from the $B A B A R$ experiment. We also measure the cross sections for the processes $e^{+} e^{-} \rightarrow K^{+}(1460) K^{-}, K_{1}^{+}(1400) K^{-}$, $K_{1}^{+}(1270) K^{-}$, and $K^{*+}(892) K^{*-}(892)$, individually, and perform a simultaneous fit on the obtained results. The fit results in a structure with mass $M=(2126.5 \pm 16.8 \pm$ 12.4) $\mathrm{MeV} / c^{2}$, width $\Gamma=(106.9 \pm 32.1 \pm 28.1) \mathrm{MeV}$, and statistical significance $6.3 \sigma$, where the uncertainties are statistical and systematic, respectively. The structure is directly produced in $e^{+} e^{-}$collisions, and thus has $J^{P C}=1^{--}$. This structure has a mass close to the masses of the vector particles $\phi(2170), \rho(2150)$, and $\omega(2290)$ listed in the PDG [1]. Its width is only consistent with the $\phi(2170)$ and is different from the others by more than $3 \sigma$.

Assuming the observed structure is $\phi(2170)$, our measurement implies that the $\phi(2170)$ has a sizable partial width to $K^{+}(1460) K^{-}, K_{1}^{+}(1400) K^{-}$, and $K_{1}^{+}(1270) K^{-}$, but a much smaller partial width to $K^{*+}(892) K^{*-}(892)$ and $K^{*+}(1410) K^{-}$. According to Ref. [10], the $3^{3} S_{1} s \bar{s}$ state mainly decays to $K^{*+}(892) K^{*-}(892)$ and $K^{*+}(1410) K^{-}$, but has a much smaller partial width to $K_{1}^{+}(1400) K^{-}$and $K^{+}(1460) K^{-}$. On the other hand, the $2^{3} D_{1} s \bar{s}$ state has an expected partial width to $K_{1}^{+}(1400) K^{-}$smaller than that to $K^{*+}(1410) K^{-}$by a factor of $2-5[10,11]$. A hybrid state is expected to decay dominantly into $K_{1}^{+}(1270) K^{-}$and $K_{1}^{+}(1400) K^{-}$, while it should be highly suppressed in the modes $K^{*+}(892) K^{*-}(892)$ and $K^{+}(1460) K^{-}$[13]. None of the above theoretical expectations are in good agreement with our experimental results.

The BESIII Collaboration thanks the staff of BEPCII and the IHEP computing center and the supercomputing center of USTC for their strong support. This work is supported in part by National Key Basic Research Program of China under Contract No. 2015CB856700; National Natural Science Foundation of China (NSFC) under Contracts No. 11625523, No. 11635010, No. 11735014 , No. 11822506, No. 11835012, No. 11961141012, No. 11335008, No. 11375170, No. 11475164, No. 11475169, No. 11625523, No. 11605196, No. 11605198, and No. 11705192; the Chinese Academy of Sciences (CAS) Large-Scale Scientific Facility Program; Joint Large-Scale Scientific Facility Funds of the NSFC and CAS under Contracts No. U1532257, No. U1532258, No. U1732263, No. U1832207, No. U1532102, No. U1732263, and No. U1832103; CAS Key Research Program of Frontier Sciences under Contracts No. QYZDJSSW-SLH003 and No. QYZDJ-SSW-SLH040; 100 Talents Program of CAS; Institute of Nuclear and Particle Physics (INPAC) and Shanghai Key Laboratory for Particle Physics and Cosmology; ERC under Contract No. 758462; German Research Foundation DFG under Contracts No. Collaborative Research Center CRC 1044 and No. FOR 2359; Istituto Nazionale di Fisica Nucleare, Italy; Ministry of Development of Turkey under Contract No. DPT2006K-120470; National Science and Technology fund; STFC (United Kingdom); The Knut and Alice Wallenberg Foundation (Sweden) under Contract No. 2016.0157; The Royal Society, UK under Contracts No. DH140054 and No. DH160214; The Swedish Research Council; U.S. Department of Energy under Contracts No. DE-FG02-05ER41374, No. DE-SC-0010118, and No. DE-SC-0012069. 
also at Ankara University,06100 Tandogan, Ankara, Turkey.

${ }^{\mathrm{b}}$ Also at Bogazici University, 34342 Istanbul, Turkey.

${ }^{c}$ Also at the Moscow Institute of Physics and Technology, Moscow 141700, Russia.

${ }^{\mathrm{d}}$ Also at the Functional Electronics Laboratory, Tomsk State University, Tomsk 634050, Russia.

eAlso at the Novosibirsk State University, Novosibirsk 630090, Russia.

${ }^{\mathrm{f}}$ Also at the NRC "Kurchatov Institute", PNPI, Gatchina 188300, Russia.

${ }^{g}$ Also at Istanbul Arel University, 34295 Istanbul, Turkey.

${ }^{\mathrm{h}}$ Also at Goethe University Frankfurt, 60323 Frankfurt am Main, Germany.

${ }^{i}$ Also at Key Laboratory for Particle Physics, Astrophysics and Cosmology, Ministry of Education; Shanghai Key Laboratory for Particle Physics and Cosmology; Institute of Nuclear and Particle Physics, Shanghai 200240, People's Republic of China.

${ }^{\mathrm{j}}$ Also at Key Laboratory of Nuclear Physics and Ion-beam Application (MOE) and Institute of Modern Physics, Fudan University, Shanghai 200443, People's Republic of China. ${ }^{\mathrm{k}}$ Also at Harvard University, Department of Physics, Cambridge, Massachusetts 02138, USA.

${ }^{1}$ Currently at: Institute of Physics and Technology, Peace Avenue 54b, Ulaanbaatar 13330, Mongolia.

${ }^{\mathrm{m}}$ Also at State Key Laboratory of Nuclear Physics and Technology, Peking University, Beijing 100871, People's Republic of China.

${ }^{\mathrm{n}}$ School of Physics and Electronics, Hunan University, Changsha 410082, China.

*Corresponding author. zhangyt2017@ustc.edu.cn

[1] M. Tanabashi et al. (Particle Data Group), Phys. Rev. D 98, 030001 (2018).

[2] B. Aubert et al. (BABAR Collaboration), Phys. Rev. D 74, 091103(R) (2006).

[3] B. Aubert et al. (BABAR Collaboration), Phys. Rev. D 76, 012008 (2007).

[4] B. Aubert et al. (BABAR Collaboration), Phys. Rev. D 86, 012008 (2012).

[5] C. P. Shen et al. (Belle Collaboration), Phys. Rev. D 80, 031101(R) (2009).

[6] M. Ablikim et al. (BES Collaboration), Phys. Rev. Lett. 100, 102003 (2008).

[7] M. Ablikim et al. (BESIII Collaboration), Phys. Rev. D 91, 052017 (2015).

[8] M. Ablikim et al. (BESIII Collaboration), Phys. Rev. D 99, 012014 (2019).

[9] T. Barnes, N. Black, and P. R. Page, Phys. Rev. D 68, 054014 (2003).

[10] G. J. Ding and M. L. Yan, Phys. Lett. B 657, 49 (2007).

[11] X. Wang, Z.-F. Sun, D.-Y. Chen, X. Liu, and T. Matsuki, Phys. Rev. D 85, 074024 (2012).

[12] S. S. Afonin and I. V. Pusenkov, Phys. Rev. D 90, 094020 (2014).

[13] G. J. Ding and M. L. Yan, Phys. Lett. B 650, 390 (2007).

[14] P. R. Page, E. S. Swanson, and A. P. Szczepaniak, Phys. Rev. D 59, 034016 (1999).
[15] Z. G. Wang, Nucl. Phys. A791, 106 (2007).

[16] H. X. Chen, X. Liu, A. Hosaka, and S.-L. Zhu, Phys. Rev. D 78, 034012 (2008).

[17] H. W. Ke and X. Q. Li, Phys. Rev. D 99, 036014 (2019).

[18] N. V. Drenska, R. Faccini, and A. D. Polosa, Phys. Lett. B 669, 160 (2008).

[19] L. Zhao, N. Li, S.-L. Zhu, and B.-S. Zou, Phys. Rev. D 87, 054034 (2013).

[20] C. Deng, J. Ping, Y. Yang, and F. Wang, Phys. Rev. D 88, 074007 (2013).

[21] Y. Dong, A. Faessler, T. Gutsche, Q. Lü, and V.E. Lyubovitskij, Phys. Rev. D 96, 074027 (2017).

[22] A. Martinez Torres, K. P. Khemchandani, L. S. Geng, M. Napsuciale, and E. Oset, Phys. Rev. D 78, 074031 (2008); S. Gomez-Avila, M. Napsuciale, and E. Oset, Phys. Rev. D 79, 034018 (2009).

[23] M. Ablikim et al. (BES Collaboration), Phys. Lett. B 685, 27 (2010).

[24] S. Godfrey and N. Isgur, Phys. Rev. D 32, 189 (1985).

[25] See Supplemental Material at http://link.aps.org/ supplemental/10.1103/PhysRevLett.124.112001 for a summary of the number of signal events, luminosity, cross section and systematic uncertainty at each energy point, the comparison of invariant mass spectra and angular distribution between data, and fit results at $\sqrt{s}=2.125$ and $2.396 \mathrm{GeV}$.

[26] M. Ablikim et al. (BESIII Collaboration), Nucl. Instrum. Methods Phys. Res., Sect. A 614, 345 (2010).

[27] S. Agostinelli et al. (GEANT4 Collaboration), Nucl. Instrum. Methods Phys. Res., Sect. A 506, 250 (2003).

[28] R. G. Ping, Chin. Phys. C 38, 083001 (2014).

[29] G. Balossini, C. M. Carloni Calame, G. Montagna, O. Nicrosini, and F. Piccinini, Nucl. Phys. B758, 227 (2006).

[30] B. Andersson and H. Hu, arXiv:hep-ph/9910285.

[31] M. Ablikim et al. (BESIII Collaboration), Chin. Phys. C 41, 063001 (2017).

[32] M. Ablikim et al. (BESIII Collaboration), Phys. Rev. D 83, 032003 (2011).

[33] N. Berger, B. J. Liu, and J. K. Wang, J. Phys. Conf. Ser. 219, 042031 (2010).

[34] B. S. Zou and D. V. Bugg, Eur. Phys. J. A 16, 537 (2003).

[35] M. Ablikim et al. (BESII Collaboration), Phys. Lett. B 598, 149 (2004).

[36] M. Ablikim et al. (BESII Collaboration), Phys. Lett. B 645, 19 (2007).

[37] F. James and M. Roos, Comput. Phys. Commun. 10, 343 (1975).

[38] E. A. Kuraev and V. S. Fadin, Yad. Fiz. 41, 733 (1985) [Sov. J. Nucl. Phys. 41, 466 (1985)].

[39] S. Actis et al., Eur. Phys. J. C 66, 585 (2010).

[40] M. Ablikim et al. (BESIII Collaboration), Chin. Phys. C 41, 063001 (2017).

[41] M. Ablikim et al. (BESIII Collaboration), Phys. Rev. D 99, 032001 (2019).

[42] M. Ablikim et al. (BESIII Collaboration), Phys. Rev. D 81, 052005 (2010).

[43] M. Ablikim et al. (BESIII Collaboration), Phys. Rev. D 87, 012002 (2013).

[44] F. von Hippel and C. Quig, Phys. Rev. D 5, 624 (1972). 